\title{
It's Not De Quervain Tenosynovitis - A Diagnosis to Consider in Persistent Wrist Pain
}

\author{
Eric Schmidt, MD, ${ }^{1}$ Yuka Kobayashi, DO, ${ }^{2}$ Andrew W. Gottschalk, MD $^{3}$ \\ ${ }^{1}$ Department of Internal Medicine, Oregon Health and Science University, Portland, OR ${ }^{2}$ Department of Family Medicine and Sports \\ Medicine, Oregon Health and Science University, Portland, OR ${ }^{3}$ Department of Orthopedics, Sports Medicine Institute, Ochsner Clinic \\ Foundation, New Orleans, LA
}

\section{CASE PRESENTATION}

A 41-year-old, right-hand-dominant racquetball player presents after 8 months of chronic, intermittent left wrist pain requiring her to take time off work and sport. She recalls mild onset of diffuse left wrist pain following long racquetball practices that gradually increased in severity and later localized to the distal dorsal radial wrist. Previous treatments included brief cessation of racquetball and activity modifications at work, wrist bracing, elbow strap, occupational therapy, nonsteroidal anti-inflammatory drugs, and heat/ice. Magnetic resonance imaging (MRI) of her wrist demonstrated no abnormal findings. Bedside ultrasound showed fluid accumulation at the area of maximal tenderness along the proximal intersection of the first and second extensor compartment tendons. She asks, "How can I return to racquetball?"

\section{BACKGROUND}

Intersection syndrome is a form of tenosynovitis involving the intersection of the musculotendinous junctions of the first and second extensor compartment tendons. The first compartment (abductor pollicis longus and extensor pollicis brevis) passes obliquely over the second compartment (extensor carpi radialis brevis and extensor carpi radialis longus). Repetitive extension-flexion of the wrist and friction at the crossover junction cause pain, swelling, and inflammation of the distal dorsal-radial forearm, classically proximal to the radial styloid. ${ }^{1}$

In the general population, the incidence of intersection syndrome ranges from $0.2 \%$ to $0.37 \% .^{2-4}$ The condition is associated with several sports, such as rowing, canoeing, racket sports, horseback riding, mountain biking, and skiing. 5,6 Typically, patients present with forearm pain, swelling, and sometimes redness and crepitus 4 to $6 \mathrm{~cm}$ proximal to the radiocarpal joint (Figure). MRI shows tendon thickening and peritendinous edema acutely, but these changes may not be seen in chronic cases because of stenosing tenosynovitis. ${ }^{3}$

\section{REVIEW OF EVIDENCE}

Treatment of intersection syndrome involves 3 escalating levels, starting with conservative management and progressing to injections and surgical intervention. ${ }^{2}$ Approximately $60 \%$ of cases will resolve with conservative management in 2 to 3 weeks. ${ }^{2,4,7-9}$
In the first phase of conservative management, the patient incorporates rest, activity modification, and immobilization with a cock-up wrist or thumb spica splint in 15-degree extension. ${ }^{2,8}$ A short course of anti-inflammatory medications (eg, ibuprofen $600 \mathrm{mg} 3$ times daily or meloxicam $7.5 \mathrm{mg}$ once daily for 7-14 days) is often necessary for pain control. Patients are also advised to use cryotherapy, elevation, compression, and taping across the dorsal forearm with force applied in the ulnar direction. ${ }^{7}$

Following the initial rest period of 2 to 3 weeks, the patient enters the second phase of conservative management that consists of gradual restoration of function and return to activity with progressive stretching, mobilization, and strength training for at least 4 to 6 weeks. ${ }^{2}$ Patients should follow the $10 \%$ rule to avoid relapse of symptoms: increase weight, repetitions, or distance by $10 \%$ per week. ${ }^{8}$

No compelling evidence-based rehabilitation protocols are available for intersection syndrome. ${ }^{1}$ If insufficient improvement is seen after phase 1 (the patient is unable to start returning to activities because of persistent symptoms) or phase 2 (the patient has a relapse of symptoms after returning to activities) of conservative management, injection therapies are offered. ${ }^{2,8}$

Several options for injection therapies are available to treat intersection syndrome. Corticosteroid injections are used most commonly, using a 1:1 mixture (approximately 0.5 to $1 \mathrm{~mL}$ ) of corticosteroid (eg, Kenalog [triamcinolone acetonide] $40 \mathrm{mg} / 1 \mathrm{~mL}$ or 3 to $6 \mathrm{mg}$ betamethasone sodium acetate) and anesthetic (eg, $1 \mathrm{~mL}$ lidocaine $1 \%$ without epinephrine) with a 1- to 1.5-inch, 22- to 25-gauge needle. ${ }^{1}$ Ultrasound-guided injections allow needle placement where the first dorsal compartment crosses over the second compartment. ${ }^{1,8}$ These injections provide resolution of symptoms within 10 days based on several case reports. ${ }^{2,5,6,8}$ The use of corticosteroid injections for de Quervain tenosynovitis (a condition similar to intersection syndrome) has level B evidence, but to our knowledge, no large series document intersection syndrome treatment outcomes in the literature. ${ }^{10-12}$ Overall risks of cortisone injections are minimal but include hypopigmentation, skin/subcutaneous fat atrophy or necrosis, tendon rupture, and infections. ${ }^{13}$

Prolotherapy is considered when the side effects of steroid therapy are a limiting factor and involves injecting a mixture of dextrose, sterile water, and lidocaine; although volumes vary, an example is $3 \mathrm{~mL}$ dextrose $50 \%, 5 \mathrm{~mL}$ saline 


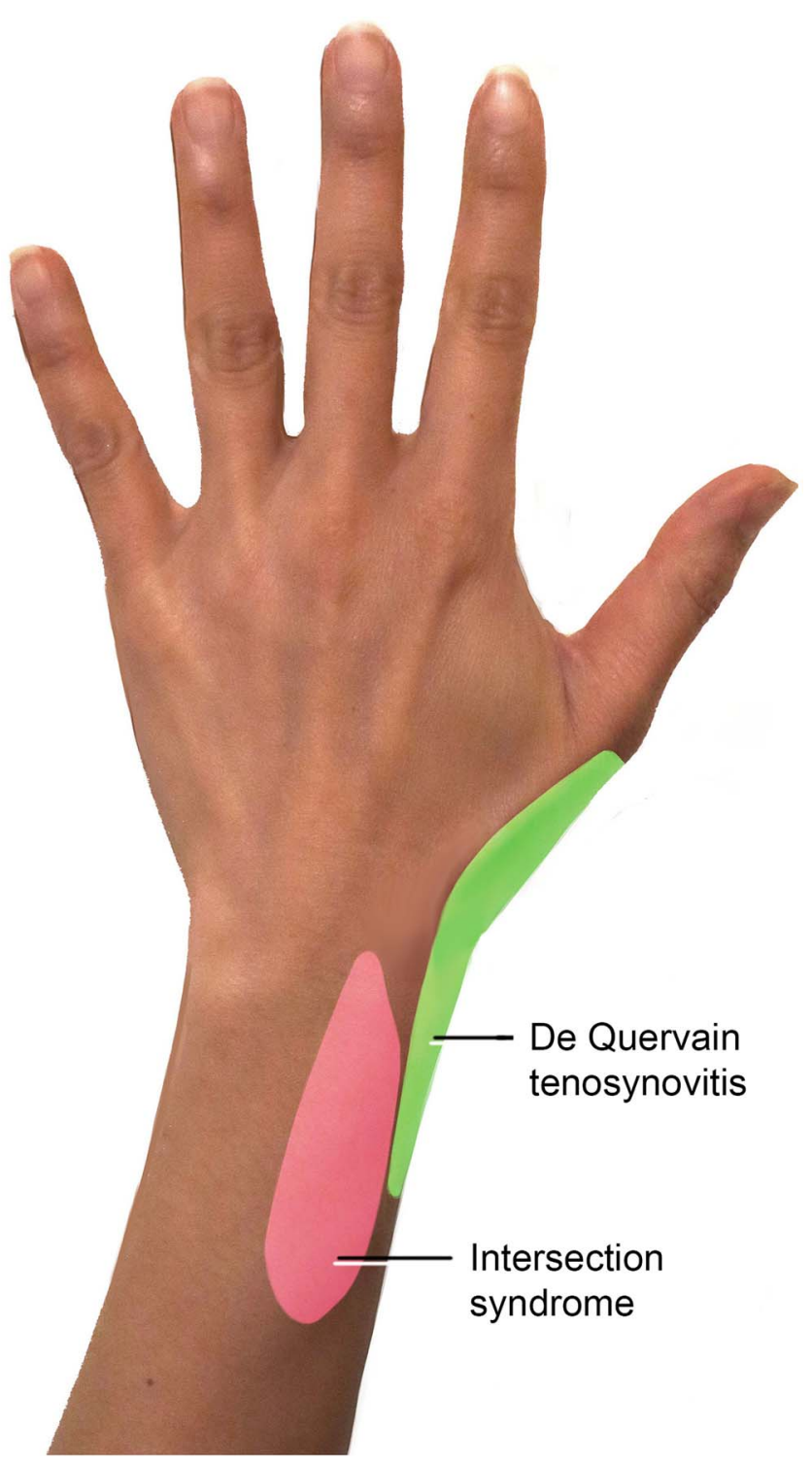

Figure. Regions of pain in De Quervain tenosynovitis and intersection syndrome.

$0.9 \%$, and $2 \mathrm{~mL}$ lidocaine $2 \% .^{1,14}$ Prolotherapy is thought to induce a proinflammatory state that triggers the release of growth factors and ultimately collagen deposition, leading to strengthening of tissue. ${ }^{1}$ As with cortisone injections, evidence to support the use of prolotherapy in treating intersection syndrome is lacking.

Hydrodissection is the injection of high volumes of saline into the compartmental space to disrupt the neurovascular bundles that grow in the tendon mechanically and reduce adhesions. ${ }^{15}$ However, no studies have been published demonstrating the effectiveness of hydrodissection for intersection syndrome.

If injections fail to resolve symptoms of intersection syndrome, surgical intervention with tenosynovectomy, longitudinal incisions of the extensor retinaculum over the second compartment, is considered. ${ }^{1}$

\section{TAKEAWAY}

Intersection syndrome is treated similarly to other types of tenosynovitis. However, more data are needed specifically regarding intersection syndrome to determine if clinical improvements are supported by the evidence.

\section{CASE RESOLUTION}

After discussion of the proposed procedure, including risks and benefits, the patient elected ultrasound-guided corticosteroid injection with $0.5 \mathrm{~mL}$ Kenalog $40 \mathrm{mg} / 1 \mathrm{~mL}$ and $0.5 \mathrm{~mL} 1 \%$ lidocaine without epinephrine. She tolerated the injection well and restarted the home exercise program that she learned in occupational therapy. At 6-week follow-up, the patient reported substantial improvement in her pain and stated that she was able to return to racquetball with minimal symptoms.

\section{ACKNOWLEDGMENTS}

The authors have no financial or proprietary interest in the subject matter of this article.

\section{REFERENCES}

1. Michols NJ, Kiel J. Intersection syndrome. StatPearls [Internet]. Updated August 12, 2020. Accessed May 25, 2021. https://www.ncbi.nlm.nih.gov/books/NBK430899/

2. Balakatounis K, Angoules AG, Angoules NA, Panagiotopoulou $\mathrm{K}$. Synthesis of evidence for the treatment of intersection syndrome. World J Orthop. 2017;8(8):619-623. doi: 10.5312/wjo.v8.i8.619

3. Costa CR, Morrison WB, Carrino JA. MRI features of intersection syndrome of the forearm. AJR Am J Roentgenol. 2003;181(5):1245-1249. doi: 10.2214/ajr.181.5.1811245

4. Descatha $A$, Leproust $H$, Roure $P$, Ronan $C$, Roquelaure $Y$. Is the intersection syndrome an occupational disease? Joint Bone Spine. 2008;75(3):329-331. doi: 10.1016/j.jbspin.2007.05.013

5. Servi JT. Wrist pain from overuse: detecting and relieving intersection syndrome. Phys Sportsmed. 1997;25(12):41-44. doi: 10.3810/psm.1997.12.1401

6. Mortalla CP, Blanco Caceres BA, Corral JB. Sindrome de la interseccion distal. Una causa poco frecuente de dolor en el antebrazo. Distal intersection syndrome: an unusual cause of forearm pain. Rheumatol Clin. 2020;16(2):128-129. doi: 10.1016/j.reumae.2018.02.002

7. Hanlon DP, Luellen JR. Intersection syndrome: a case report and review of the literature. J Emerg Med. 1999;17(6):969-971. doi: 10.1016/s0736-4679(99)00125-0

8. Yonnet JG. Intersection syndrome in a handcyclist: case report and literature review. Top Spinal Cord Inj Rehabil. 2013;19(3):236-243.

9. Kaneko S, Takasaki H. Forearm pain, diagnosed as intersection syndrome, managed by taping: a case series. J Orthop Sports Phys Ther. 2011;41(7):514-519. doi: 10.2519/jospt.2011.3569

10. Lane LB, Boretz RS, Stuchin SA. Treatment of de Quervain's disease: role of conservative management. J Hand Surg Br. 2001;26(3):258-260. doi: 10.1054/jhsb.2001.0568

11. Richie CA III, Briner WW Jr. Corticosteroid injection for treatment of de Quervain's tenosynovitis: a pooled quantitative literature evaluation. J Am Board Fam Pract. 2003;16(2):102-106. doi: 10.3122/jabfm.16.2.102

12. Stephens MB, Beutler Al, O'Connor FG. Musculoskeletal injections: a review of the evidence. Am Fam Physician. 2008;78(8):971-976. 
13. Brinks A, Koes BW, Volkers ACW, Verhaar JAN, Bierma-Zeinstra SMA. Adverse effects of extra-articular corticosteroid injections: a systematic review. BMC Musculoskelet Disord. 2010;11:206. doi: 10.1186/1471-2474-11-206

14. Scarpone M, Rabago DP, Zgierska A, Arbogast G, Snell E. The efficacy of prolotherapy for lateral epicondylosis: a pilot study.
Clin J Sport Med. 2008;18(3):248-254. doi: 10.1097/JSM.0b013e318170fc87

15. Burke CJ, Adler RS. Ultrasound-guided percutaneous tendon treatments. AJR Am J Roentgenol. 2016;207(3):495-506. doi: 10.2214/AJR.16.16089

This article meets the Accreditation Council for Graduate Medical Education and the American Board of Medical Specialties Maintenance of Certification competencies for Patient Care and Medical Knowledge.

(C2021 by the author(s); licensee Ochsner Journal, Ochsner Clinic Foundation, New Orleans, LA. This article is an open (c) (i) access article distributed under the terms and conditions of the Creative Commons Attribution (CC BY) license (creativecommons.org/licenses/by/4.0/legalcode) that permits unrestricted use, distribution, and reproduction in any medium, provided the original author(s) and source are credited. 